\title{
A constitutive analysis of the extensional flows of nearly monodisperse polyisoprene melts
}

\section{Rasmussen, Henrik K.}

Published in:

Polymer

Link to article, DOI:

10.1016/j.polymer.2016.05.019

Publication date:

2016

Document Version

Peer reviewed version

Link back to DTU Orbit

Citation (APA):

Rasmussen, H. K. (2016). A constitutive analysis of the extensional flows of nearly monodisperse polyisoprene melts. Polymer, 104, 251-257. https://doi.org/10.1016/j.polymer.2016.05.019

\section{General rights}

Copyright and moral rights for the publications made accessible in the public portal are retained by the authors and/or other copyright owners and it is a condition of accessing publications that users recognise and abide by the legal requirements associated with these rights.

- Users may download and print one copy of any publication from the public portal for the purpose of private study or research.

- You may not further distribute the material or use it for any profit-making activity or commercial gain

- You may freely distribute the URL identifying the publication in the public portal

If you believe that this document breaches copyright please contact us providing details, and we will remove access to the work immediately and investigate your claim. 


\title{
A constitutive analysis of the extensional flows of nearly monodisperse polyisoprene melts
}

\author{
Henrik Koblitz Rasmussen \\ Department of Mechanical Engineering \\ Technical University of Denmark, DK-2800 Kgs. Lyngby, Denmark
}

\begin{abstract}
Here two particular formulations [27, 28, 38] of the 'interchain pressure' [39], incorporated into the molecular stress function method [27], are used to assess the extensional [22, 25] and shear viscosities [40] of narrow molecular weight distributed (NMMD) polyisoprene melts. These two formulations are expected to represent the highest [27, 38] and lowest level [28] of the 'interchain pressure'. The needed Rouse times are here defined as $\tau_{R} / \tau_{\max } \propto\left(M / M_{e}\right)^{-1.4}$ with a proportional factor of 1.4 , achieved based on the viscosity measurement. $\tau_{\max }$ is the maximal relaxation time, $M$ the molecular weight and the entanglement molecular weight $M_{e}=$ $(4 / 5) \varrho R T / G_{N}^{0}[39]$. $\varrho$ is the density, $R$ the gas constant, $T$ the temperature and $G_{N}^{0}$ the plateau modulus. The method by $[27,38]$ predicts start-up of extensional viscosities significantly below the measured value. The formulations by [28] seem to be in agreement with both the start-up of extension as well as the shear flow of all NMMD polyisoprenes. Potential non-isothermal effects were addressed computationally using the pseudo time principle, assuming the most critical case of adiabatic heating.
\end{abstract}

\section{Introduction}

The idea of universality in the flow dynamics of entangled monodisperse polymer systems is appealing. Especially for the linear viscoelasticity (LVE), experimental and theoretical investigations have shown a unique dependence on the entanglement molecular weight, $M_{e}$. For polymers with a molar mass, $M$, above the entanglement molecular weight, the zero shear viscosity scales as $\left(M / M_{e}\right)^{3.4}[1]$ and each type of polymer has a well-defined plateau modulus [2] independent of length and molecular distribution. For entangled monodisperse polymer systems, theoretical results, initiated by Doi and Edwards [3], agree with experimental findings. The concept of contour length fluctuations [4] was the first to explain the observed molar mass dependence in the power of 3.4 [5], but the area still evolves in its basic theoretical understanding [6].

The idea of universality in the flow dynamics of entangled polymer systems was a guideline for the theoretical considerations in the earliest tube theories $[7,8,9,10]$. It was challenged by the extension measurements of the start-up and steady extensional viscosities on solutions of narrow molecular weight distributed (NMMD) polymers and pure melts, respectively. These ruled out the possibility of a unified flow physics of melts and solutions based on entanglements. It motivated further theoretical developments $[11,12,13]$ in an effort to create a unified theoretical framework. Recently, to investigate the particular consequences of the presence of the solvent, Huang et al. [14, 15] made start-up and steady extension measurements on the most ideal dilutions: NMMD polystyrenes in their own styrene oligomer. The results were surprising. The measurements for diluents consisting of chains, in term of Kuhn steps, were in-between pure melts and corresponding solutions [16], all with 
Despite the differences between melts and solutions, there seems to be a remarkable amount of experimental evidence, in shear as well as extension, of a correlation between the entanglement number and the time constant controlling the transition to the strain-hardening flow. For entangled monodisperse polymer systems the strain-hardening viscoelasticity seems to be controlled by a single time scale, commonly referred to as the Rouse time $\tau_{R}$. It defines the transition from a pure configurational stress on large time scales [3] to a strain-hardening regime at small times [8, 17, 18, 19, 20].

Keeping the above efforts in mind, the first attempt to measure extensional viscosities on nearly monodisperse polymer systems was actually done previous to all of the above references, considering monodisperse systems. In the mid-seventies Vinogradov and co-workers [21] extended narrow molecular weight distributed (NMMD) polyisoprene uniaxially. The theoretical importance of their work was limited, probably due to low extensional strain values where only a few reached a value of 2. Notice that the extensional strain is defined as $\epsilon(t)=\ln (l(t) / l(0))$, where $l(t)$ and $l(t=0)$ are the distances between two particles in the direction of the extension at time $t$ and $t=0$, respectively. Within the recent years, new attempts to measure the extensional viscosities on NMMD polyisoprene have been made $[22,23,24,25]$. The purpose of this paper is to find out how these measurements relate to experimental rheometric investigation on other entangled polymer systems and recent theoretical ideas, both being unclear.

\section{Non-isothermal extension}

As the quantitative method to handle different underlying physical mechanisms the 'molecular stretch' representation from Wagner [26] will be applied. Its general functional representation seems to be a sufficiently accurate basis for the fluid mechanics of MNND polymers [27] and their solutions [28], although the actual underlying physical mechanisms are debated [26, 28]. This 'molecular stretch' method is not unique. Other frameworks are likely to have an equal validity [29, 30].

The 'molecular stretch' representation is formulated isothermally. As discussed later, the issue of potential non-isothermability during extension of NMMD melts may need to be addressed due to the heat of work. The well-established time-temperature superposition is only valid during isothermal conditions. The changing temperature during the stress build up will need a more comprehensive method. The experimental evidence is limited. Without considering specific constitutive equations only one paper has been published by $\mathrm{Yu}$ et al. [31]. Previous studies [32, 33, 34] were all based on the assumption of time and strain separability. This is only a correct assumption for small, i.e. linear, deformations.

For linear conditions Morland and Lee [35] introduced the 'pseudo time' principle, considering a 'thermo-rheological simple' material. The 'pseudo time', $\xi\left(\boldsymbol{x}, t, t^{\prime}\right)$, at the past time $t^{\prime}$ relatively to the present time $t$ is defined as

$$
\xi^{\prime} \equiv \xi\left(\boldsymbol{x}, t, t^{\prime}\right)=\int_{t^{\prime}}^{t} \frac{1}{a_{T}\left(T\left(\boldsymbol{x}, t, t^{\prime \prime}\right)\right)} d t^{\prime \prime},
$$

where $a_{T}$ is the classical time-temperature superposition shift factor, calculated at the temperature, $T\left(\boldsymbol{x}, t, t^{\prime \prime}\right)$, at the past time, $t^{\prime \prime} . a_{T}$ is evaluated relative to the fixed temperature $T_{0}$. The 'pseudo time' should be evaluated on all particle paths. Mathematically the reference state is the (Cartesian) particle coordinates $\left(x_{1}^{\prime}, x_{2}^{\prime}, x_{3}^{\prime}\right)$ at the past time state displaced to the coordinates $\left(x_{1}, x_{2}, x_{3}\right)$ at the present time. Notice $\partial \xi^{\prime} / \partial t^{\prime}=-1 / a_{T}\left(T^{\prime}\right)$ and $\partial \xi^{\prime} / \partial t=1 / a_{T}\left(T^{\prime}\right)$. The latter one evaluated at the present time where $t^{\prime}=t$ is $\partial \xi / \partial t=1 / a_{T}(T)$. A short notation for the present and past temperatures has been used, referring to the present particle $\boldsymbol{x}$, as $T=T(\boldsymbol{x}, t, t)$ and $T^{\prime}=T\left(\boldsymbol{x}, t, t^{\prime}\right)$, respectively. 
'thermo-rheological simple' even at high strain values. The 'molecular stretch' representation of the stress tensor $\sigma_{\mathrm{ij}}$ in a non-isothermal representation based on the pseudo time is given as

$$
\sigma_{i j}=\frac{\varrho T}{\varrho_{0} T_{0}} \int_{0}^{\infty} M\left(\xi\left(\boldsymbol{x}, t, t^{\prime}\right)\right) f\left(\boldsymbol{x}, t, t^{\prime}\right)^{2} 5\left\langle\frac{\left[\mathbf{E}\left(\boldsymbol{x}, t, t^{\prime}\right) \cdot \mathbf{u}\right]\left[\mathbf{E}\left(\boldsymbol{x}, t, t^{\prime}\right) \cdot \mathbf{u}\right]}{\left|\mathbf{E}\left(\boldsymbol{x}, t, t^{\prime}\right) \cdot \mathbf{u}\right|^{2}}\right\rangle d \xi^{\prime} .
$$

The initial value of $f\left(\boldsymbol{x}, t^{\prime}, t^{\prime}\right)=1$ and $M(\ldots)$ represents the memory function determined at the fixed temperature $T_{0}$. Notice that all stresses are temperature corrected to the present temperature, $T$, using the ratio $\varrho_{0} T_{0} /(\varrho T)$ where $\varrho_{0}$ is the density at the fixed temperature $T_{0}$ and $\varrho$ is the density at the present temperature $T$. All the angular brackets are unit sphere integrals defined as $\langle\ldots\rangle=1 /(4 \pi) \int_{|\mathbf{u}|=1} \ldots d \mathbf{u}$ where $\mathbf{u}$ is a unit vector. The components of the displacement gradient tensor $\mathbf{E}\left(\boldsymbol{x}, t, t^{\prime}\right)$ are $E_{\mathrm{ij}}\left(\boldsymbol{x}, t, t^{\prime}\right)=\partial x_{\mathrm{i}} / \partial x_{\mathrm{j}}{ }^{\prime}, i=1,2,3$ and $j=1,2,3$. To integrate equation (2) in real time $\partial \xi^{\prime} / \partial t^{\prime}=-1 / a_{T}\left(T^{\prime}\right)$ is inserted.

$$
\sigma_{i j}=\frac{\varrho T}{\varrho_{0} T_{0}} \int_{-\infty}^{t} M\left(\xi\left(\boldsymbol{x}, t, t^{\prime}\right)\right) f\left(\boldsymbol{x}, t, t^{\prime}\right)^{2} 5\left\langle\frac{\left[\mathbf{E}\left(\boldsymbol{x}, t, t^{\prime}\right) \cdot \mathbf{u}\right]\left[\mathbf{E}\left(\boldsymbol{x}, t, t^{\prime}\right) \cdot \mathbf{u}\right]}{\left|\mathbf{E}\left(\boldsymbol{x}, t, t^{\prime}\right) \cdot \mathbf{u}\right|^{2}}\right\rangle \frac{1}{a_{T}\left(T\left(\boldsymbol{x}, t, t^{\prime}\right)\right)} d t^{\prime} .
$$

The 'stretch evolution' equation for, ideally, monodisperse linear polymers in a non-isothermal as well as generic formulation is

$$
\frac{\partial}{\partial \xi} f\left(\boldsymbol{x}, t, t^{\prime}\right)=f\left(\boldsymbol{x}, t, t^{\prime}\right) \frac{\partial}{\partial \xi}\left\langle\ln \left|\mathbf{E}\left(\boldsymbol{x}, t, t^{\prime}\right) \cdot \mathbf{u}\right|\right\rangle-\frac{g\left(f\left(\boldsymbol{x}, t, t^{\prime}\right)\right)}{\tau_{R}} .
$$

$g$ is a non-negative function where $g(1)=0$ and $d g(f) / d f=1$ for $f=1$. $\tau_{R}$ is a time constant, referred to as the Rouse time, determined at the fixed temperature $T_{0}$. In the present model this is scaled based on the equality $d g(f) / d f=1$ for $f=1 . g$ is currently undefined but will be defined explicitly later on. Alternatively, equation (4) can be written in the real time

$$
\frac{\partial}{\partial t} f\left(\boldsymbol{x}, t, t^{\prime}\right)=f\left(\boldsymbol{x}, t, t^{\prime}\right) \frac{\partial}{\partial t}\left\langle\ln \left|\mathbf{E}\left(\boldsymbol{x}, t, t^{\prime}\right) \cdot \mathbf{u}\right|\right\rangle-\frac{g\left(f\left(\boldsymbol{x}, t, t^{\prime}\right)\right)}{\tau_{R} \cdot a_{T}(T(\boldsymbol{x}, t, t))}
$$

as $\partial \xi / \partial t=1 / a_{T}(T)$ evaluated at $t^{\prime}=t$

\section{Entanglements}

Currently it seems that no published constitutive model is quantitatively able to predict both the linear and nonlinear flow dynamics of NMMD polymer melt systems, within the same theoretical framework. At least not experimentally validated ones $[24,26]$. The present study is no exception. The linear dynamics is characterized by a maximal relaxation time, $\tau_{\max }$. The maximal relaxation time follows the well-established relation of $\tau_{\max } \propto\left(M / M_{e}\right)^{3.4}[5]$. One approach to determine the linear viscoelastic dynamics of NMMD polymers accurately is the method by Baumgaertel, Schausberg and Winter (BSW) [36, 37]. It is the one used here. The memory function is

$$
\begin{aligned}
M\left(t-t^{\prime}\right) & =\int_{0}^{\infty} \frac{H(\tau)}{\tau^{2}} e^{\left(-\left(t-t^{\prime}\right) / \tau\right)} d \tau \\
H(\tau) & =n_{e} G_{N}^{0}\left[\left(\frac{\tau}{\tau_{\max }}\right)^{n_{e}}+\left(\frac{\tau}{\tau_{c}}\right)^{-n_{g}}\right] h\left(1-\tau / \tau_{\max }\right) .
\end{aligned}
$$


plateau modulus. All time constants, of course, change with the temperature according to the timetemperature superposition principle.

The vast majority of different theoretical concepts $[8,9,17,18,19,20,27,38]$ suggest the existence of a characteristic time constant for the transition to a strain hardening regime for fast flows. It is observed experimentally particularly for NMMD polymers and solutions, both in shear and extension. Most of these theoretical approaches expect a dependence of this time constant as the square of the number of entanglements, i.e. $\left(M / M_{e}\right)^{2}$.

With an expected proportionality to the entanglement number of $\tau_{R} \propto\left(M / M_{e}\right)^{2}$ and a maximal relaxation time following $\tau_{\max } \propto\left(M / M_{e}\right)^{3.4}$ the ratio between these is $\tau_{R} / \tau_{\max } \propto\left(M / M_{e}\right)^{-1.4}$. For a particular polymer $M_{e}=(4 / 5) \varrho R T / G_{N}^{0}$, as derived by Doi and Edwards [39], is used. This relation is particularly important for polyisoprenes. Notice that $R$ is the gas constant.

The composition of the polyisoprene backbone differs highly in the published studies. Especially the amount of 3,4 units compared to 1,4 units seems to affect the linear viscoelastic parameters significantly. A dominant quantity of 3,4 units increases the measured plateau modulus significantly. The quantity was reported to be about $60 \%$ in the work by Liu et al. [25] whereas the similar quantities were about $5-6 \%$ and $8.6 \%$ in Auhl et al. [40] and Nielsen et al. [22], respectively. The remaining units were cis- and trans-1,4 units. In all the latter references a value of $G_{N}^{0}=476 \mathrm{kPa}$ at $25^{\circ} \mathrm{C}$ was experimentally determined for all compositions. Further, the $\tau_{c}$ values were the same.

The needed BSW parameters are calculated by data fittings to the BSW model (7) using the method from Rasmussen et al. [41] and the obtained values can be found in table 1. For all involved polyisoprenes it is possible to use the fixed values of $n_{e}=0.22$ and $n_{g}=0.65$. The measured mechanical spectroscopic data from Liu et al. [25] and Nielsen et al. [22] with the actual fittings are shown in figure 1. Of importance $G_{N}^{0}=380 \mathrm{kPa}\left(\right.$ at $\left.25^{\circ} \mathrm{C}\right)$ for the polyisoprene from Liu et al. [25].

In some cases a more simplified method can be applied, in the case where $\tau_{c}$ and $G_{N}^{0}$ are known for the material. $\tau_{\max }$ can be calculated from the zero shear viscosity given as

$$
\eta_{0}=\int_{0}^{\infty} G(s) d s=n_{e} G_{N}^{0} \tau_{\max }\left(\frac{1}{1+n_{e}}+\frac{1}{1-n_{g}}\left(\frac{\tau_{c}}{\tau_{\max }}\right)^{n_{g}}\right) .
$$

This equation is used where accurate zero shear viscosities are available [40].

\section{Interchain tube pressure}

The current theoretical effort considering NMMD linear polymers melts has been based almost only on monodisperse polystyrenes $[42,43]$. The exception is the NMMD polyisoprene in start-up of shear flow [40]. In contrast to extension flow nonlinearly shear measurements are not particularly sensitive to changes in material properties.

One tube theory able to handle the MNND polystyrene measurements quantitatively [38] was based on the interchain pressure [39]. The random motion of the polymer chains, in term of Kuhn chains, imposes a thermal pressure on the tube wall. Doi and Edwards [39] derived the well-established relation for the thermal pressure

$$
p=\frac{\pi^{2}}{3} \frac{N b^{2}}{L_{V}^{2}} \frac{k T}{V} .
$$

$T$ is the temperature, $k$ Boltzmann constant, where $k T$ represents the thermal energy. $N$ is the number of Kuhn segments in the short chain, where $b$ is the length of these segments. $V$ is the volume of the tube, where $L_{V}$ is its length dimension. 
pressure seems unnecessary to explain the extensional flow properties. A straightforward classical Rouse type of model is sufficient. Actually, recently Wagner [26] suggested a unified model for all types of NMMD polymer systems: The lowering of the glass transition temperature from the pure melt state, due to the presence of the solvent, changed the weighting between a pure Rouse dynamic and the changing interchain pressure [38] as presented by Wagner et al. [27].

As the 'molecular stretch' representation has been used in the equation (5), the stress is not sensitive to the particular choice of model, melt or diluted, at low extensional strain values. These are values until a strain in-between 1 and 1.5. Notice that the real kinematic strain is defined as $\epsilon(t)=\ln (l(t) / l(0))$, where $l(t)$ and $l(t=0)$ are the (narrow) distances between two particles in the direction of the extension. Here there is not distinguished between the real strain and the set strain on the particular rheometer. Although one issue is the actual value of the proportionality factor controlling the Rouse time, of course relative to the maximal relaxation time, which depends on the used theoretical approach. The referred measurements in extension are all performed on a Sentmanat extensional rheometer (SER) [44], and the expected deviations from an ideal uniaxial deformation are well within the experimental scattering [45]. Figure 2 and 3A shows the startup of extensional viscosity, $\bar{\eta}^{+}$, together with the original 'molecular stretch' representation of the interchain pressure [27] where $g(f)=f\left(f^{3}-1\right) / 3$ in equation (5). These are the dotted $(\cdots)$ lines. The most optimal proportionality for the Rouse time has been obtained based on these data as $\tau_{R} / \tau_{\max }=1.4\left(M / M_{e}\right)^{-1.4}$. Figure 2 contains the polyisoprene measurements from Liu et al. [25] and figure 3 the data from Nielsen et al. [22], with a polymer containing 75 and 344 entanglements, respectively. Notice that the start-up (at time $t=0$ ) of extension viscosity is defined as $\bar{\eta}^{+}=$ $\left(\sigma_{33}-\sigma_{11}\right) / \dot{\epsilon}$. The relation between the extensional rate, $\dot{\epsilon}$, and strain, $\epsilon(t)$, is given as $\epsilon(t)=\dot{\epsilon} \cdot t$, where the start-up of the extension is at the time $t=0$.

The 'molecular stretch' representation of the interchain pressure (Marrucci and Ianniruberto [38]) using $g(f)=f\left(f^{3}-1\right) / 3$ gives quantitative agreement with the extensional dynamics [27] and shear [46] for a broad range of molecular weight NMMD polystyrene melts. For polyisoprenes this is not the case. In figure 2 and particularly in figure 3A the measurements are severely above the model prediction. It requires an extensional strain of about 2.5 to be able to distinguish between the model based on NMMD polystyrene melts flow and the measurements. Notice that the non-isothermal effect is not included, because it only has a minor effect by lowering the calculations slightly, resulting in an increase in the difference between the interchain pressure model by Wagner et al. [27] and the data.

Consequently, there does not seem to be a universality of the flow physics in-between ideally monodisperse but different polymer melts.

\section{Constant interchain tube pressure}

Particularly for polyisoprene, a potential alternative explanation is the one published recently by Rasmussen [47]. Here the interchain pressure idea is used to explain quantitatively the flow dynamics of bi-disperse polyisoprenes and polystyrenes polymer melts. They followed the idea of a constant interchain pressure, originally introduced by Rasmussen and Huang [28], to explain the flow dynamics of oligomer diluted polystyrene. Basically a Kuhn chain in a random state will impose a constant thermal pressure [39] on the wall of the tube. This requires that the surrounding medium are chains. They should have a length of at least two Kuhn steps. This idea was extended to bi-disperse systems where the short chain was long enough to be entangled, but still in a random state. It seems that a random state reduces the interchain pressure to the lowest possible value, which is a constant 
highest possible level of strain hardening, where idea from Marrucci and Ianniruberto [38] seems to represent the lowest level.

If the constant interchain pressure idea is applied to the NMMD polyisoprene the maximal extensibility needs to be addressed. Rasmussen and Huang [28] used the number of Kuhn steps in an entanglement strand, $N_{e}$, for this extensibility. The SER equipment can measure at a maximal strain of 3.8, corresponding to an extension of a factor $\exp (3.8)=44.7$, and the measurements from Liu et al. [25] have a maximal strain of 2.6 or a relative extension of a factor of $\exp (2.6)=13.5$. The different structural configurations in polyisoprene have a large impact on the value of $N_{e}$. A value of about $50[48,49]$ would be expected for the polyisoprene in Nielsen et al. [22], where in the PI melt in the work by Liu et al. [25] it is above 15 [49]. As both extension values stay below the maximal extensibility it has not been included in the models. The constant interchain pressure, without the maximal extensibility, is mathematically defined with a function $g(f)=f(f-1)$ [28]. If this is applied to the NMMD polyisoprene in figure 2 and $3 \mathrm{~B}$, the results are surprising. The constant interchain pressure modelling are the solid (-) lines. The difference between the measured elongational stresses by Liu et al. [25] and Nielsen et al. [22], and the calculated are maximal about $20 \%$ for most of the measurements in the data sets. One of the data sets in figure 2 though locally displays a larger deviation of about $35 \%$. The modelling is isothermal. This seems to be an acceptable assumption concerning the measurements from Liu et al. [25], as the calculated stress decrease is theoretically expected to be less than 10\%. The measurements in Nielsen et al. [22] are of higher strain, stresses and rates. It follows that the non-isothermability is not likely to be an acceptable assumption at the highest stress values, as discussed in details in Nielsen et al. [22].

The maximal possible non-isothermal effect is obtained considering the adiabatic condition during the extension, and assuming that the stress is of pure entropic origin. The temperature increase from the heat of work is then given as $T-T_{0}=\int_{0}^{\epsilon}\left(\sigma_{33}-\sigma_{11}\right) d \epsilon^{\prime} /\left(\varrho C_{p}\right)$. The values of the specific heat capacity of $C_{p}=1930 \mathrm{~J} /(\mathrm{kg} \mathrm{K})[22]$ and the density of $\varrho=913 \mathrm{~kg} / \mathrm{m}^{3}$ [40] has been used for the polyisoprene. The effect of the adiabatic temperature increase has been added in figure 3B. It has a severe impact on the viscosity calculations at high strain values. Until actual values of the temperature increase are known, i.e. measured, it is difficult to give an accurate theoretical evaluation of the non-isothermal effect, particularly considering the high strain values.

Any constitutive equation needs to be valid not only in extension, but also in shear. For NMMD polyisoprenes Auhl et al. [40] presented the start-up of shear flow measurements for a range of different molecular weights, with entanglement numbers ranging from 3.5 to 59.4. These four sets of start-up of shear viscosities, $\eta^{+}$, are shown in figure 4A-D. The calculated shear viscosities, corresponding to the experiments, have been inserted in figure 4A-D. The difference between the measured and calculated shear viscosities are maximal about $20 \%$ for most of the data. The exception is the viscosities for the PI-90k melt in figure 4B, where the measurement at the three highest shear rates displays deviations as large as of about $40 \%$ locally. The used Rouse time has been marked on the figures showing that the transition between the pure configurational flow and the strain hardening flow is well represented.

\section{Summary and conclusions}

The available start-up of extension and shear for a wide range of molecular weights (and compositions) polyisoprenes have been compared with the current theories based on the 'interchain pressure' [39]. The entanglement numbers range from 3.5 to 344 . Here two particular formulations of the 'interchain pressure' were incorporated into the molecular stress function method [27] allowing a quantitative 
effect of the reduction of the tube of the surrounding polymers. The second one assumes the lowest value of the interchain pressure, a constant value, from Rasmussen and Huang [28].

The Marrucci and Ianniruberto [38] suggestion for the 'interchain pressure' has been able to model accurately the flow dynamics of a broad range of NMMD polystyrene melts. But it is incapable of predicting the extensional dynamics of NMMD polyisoprenes. The predicted viscosities are significantly below the measured value. There does not seem to be a universal flow physics in-between ideally monodisperse polymer melts. The lack of universality of monodisperse polymer melt has been addresses previously. Masubuchi et al. [50] were unable to display the extensional strain hardening, similar to the PI melts, in a NMMD poly n-butyl acrylate melt [51] if their model [50] were able to predict the flow behaviour of NMMD polystyrene melt. The model by Masubuchi et al. [Masubuchi et al. 2014] was based on the monomeric friction idea.

The constant 'interchain pressure' (CIP) model by Rasmussen and Huang [28] was originally suggested for bi-disperse systems containing short polymer chains (i.e. longer than two Kuhn step) in a random state in long chains. It has been able to handle the flow dynamics of a broad range of bi-disperse polystyrenes and polyisoprenes. Moreover, it has been shown here that this model is also in agreement with both the start-up of extension as well as the shear flow of the NMMD polyisoprenes.

The non-isothermal effect has been addressed theoretically by using the pseudo time principle, to evaluate potential non-isothermal effects.

Table 1: Polyisoprene melt parameters at $25^{\circ} \mathrm{C}$. The values of $n_{e}=0.22$ and $n_{g}=0.65$ have been used for all the polyisoprenes in the table. The $Z=M_{w} / M_{e}$ where $M_{e}=(4 / 5) \varrho R T / G_{N}^{0}$ and $\varrho=0.913$ $\mathrm{g} / \mathrm{cm}^{3}$ [40]. $M_{w}$ and $M_{n}$ are the weight and molar mass average molecular weight, respectively.

\begin{tabular}{l|rrrrrrr}
\hline Name & Ref. & $M_{w}$ & $M_{w} / M_{n}$ & $G_{N}^{0}$ & $\eta_{0}$ & $\tau_{\max }$ & $\tau_{c}$ \\
PI-12k & {$[40]$} & $13.5 \mathrm{~kg} /$ mole & 1.04 & $476 \mathrm{kPa}$ & $26.4 \mathrm{Pas}$ & $0.000191407 \mathrm{~s}$ & $13 \mu \mathrm{s}$ \\
PI-30k & {$[40]$} & $33.6 \mathrm{~kg} /$ mole & 1.03 & $476 \mathrm{kPa}$ & $448 \mathrm{Pas}$ & $0.00485897 \mathrm{~s}$ & $13 \mu \mathrm{s}$ \\
PI-90k & {$[40]$} & $94.9 \mathrm{~kg} /$ mole & 1.03 & $476 \mathrm{kPa}$ & $21500 \mathrm{Pas}$ & $0.249048 \mathrm{~s}$ & $13 \mu \mathrm{s}$ \\
PI-200k & {$[40]$} & $225.9 \mathrm{~kg} /$ mole & 1.03 & $476 \mathrm{kPa}$ & $390000 \mathrm{Pas}$ & $4.53960 \mathrm{~s}$ & $13 \mu \mathrm{s}$ \\
& {$[22]$} & $1310 \mathrm{~kg} /$ mole & 1.08 & $476 \mathrm{kPa}$ & - & $2700 \mathrm{~s}$ & $13 \mu \mathrm{s}$ \\
& {$[25]$} & $356 \mathrm{~kg} /$ mole & 1.1 & $380 \mathrm{kPa}$ & - & $7000 \mathrm{~s}$ & $7 \mathrm{~ms}$
\end{tabular}


Figure 1: Loss, $G^{\prime \prime}$ (open circles and diamonds; $\circ$ and $\left.\diamond\right)$ and storage moduli, $G^{\prime}$ (bullets and squares; - and $\mathbf{\square}$ ), both as a function of the angular frequency $\omega$ at $25^{\circ} \mathrm{C}$. $\circ$ and $\bullet$ are the $G^{\prime \prime}$ and $G^{\prime}$ values, respectively, of the $356 \mathrm{~kg} /$ mole NMMD polyisoprene mesured by Liu et al. [25]. The $\diamond$ and $\mathbf{\square}$ are the $G^{\prime \prime}$ and $G^{\prime}$ values, respectively, and the for the $1310 \mathrm{~kg} /$ mole NMMD polyisoprene measured by Nielsen et al. [22].

Figure 2: The startup of extensional viscosity, $\bar{\eta}^{+}$for the $356 \mathrm{~kg} /$ mole NMMD polyisoprene measured by Liu et al. [25], as a function of the extensional strain, $\epsilon$, measured at $25^{\circ} \mathrm{C}$. The extension rates, $\dot{\epsilon}^{+}$, are $1 s^{-1}, 0.3 s^{-1}, 0.1 s^{-1}, 0.03 s^{-1}, 0.01 s^{-1}, 0.003 s^{-1}$ and $0.001 s^{-1}$ from the top to the bottom data series. The dotted lines $(\cdots)$ are the corresponding isothermal predictions to the data from the equation (5) where $g(f)=f\left(f^{3}-1\right) / 3$ [27]. The solid lines (-) are the corresponding isothermal predictions to the data from the equation (5) where $g(f)=f(f-1)$ [28]. The computations are based on the parameters listed in table 1.

Figure 3: The startup of extensional viscosity, $\bar{\eta}^{+}$for the $1310 \mathrm{~kg} /$ mole NMMD polyisoprene measured by Nielsen et al. [22], as a function of the extensional strain, $\epsilon$, measured at $25^{\circ} \mathrm{C}$. The extension rates, $\dot{\epsilon}^{+}$, are $5 s^{-1}, 3.5 s^{-1}, 2 s^{-1}, 1 s^{-1}, 0.6 s^{-1}, 0.2 s^{-1}, 0.06 s^{-1}$ and $0.003 s^{-1}$ from the top to the bottom data series. The computations are based on the parameters listed in table 1. (A) The dotted lines $(\cdots)$ are the corresponding isothermal predictions to the data from the equations (5) where $g(f)=f\left(f^{3}-1\right) / 3[27]$. (B) The solid lines $(-)$ are the corresponding isothermal predictions to the data from the equation (5) where $g(f)=f(f-1)$ [28]. (B) The dashed lines (- - -) are the corresponding non-isothermal predictions to the data from the equation (5) where $g(f)=f(f-1)$ [28] based on the adiabatic condition.

Figure 4: The startup of shear viscosity $\eta^{+}$as a function of the time $t$ for the NMMD polyisoprenes measured by Auhl et al. [40] at $-35^{\circ} \mathrm{C}$. All details concerning the time-temperature superposition shifting can be found in Auhl et al. [40]. The shear rates, $\dot{\gamma}$, are listed in the figures. (A) contains for the shear viscosities for the $225.9 \mathrm{~kg} /$ mole NMMD polyisoprene (PI-200k), (B) contains for the shear viscosities for the $94.9 \mathrm{~kg} /$ mole NMMD polyisoprene (PI-90k). (C) contains for the shear viscosities for the $33.6 \mathrm{~kg} /$ mole NMMD polyisoprene (PI-30k), and (D) contains for the shear viscosities for the $13.5 \mathrm{~kg} /$ mole NMMD polyisoprene (PI-12k). The solid lines (-) are the corresponding isothermal predictions to the data from the equation (5) where $g(f)=f(f-1)$ [28]. The computations are based on the parameters listed in table 1 . The dotted line $(\cdots)$ is the linear viscoelastic predictions based on the parameters listed in table 1 using equation (7). 
[1] G.C. Berry, T.G. Fox, Advances in Polymer Science 5 (3) (1968) 261-357.

[2] S. Onogi, T. Masuda, K. Kitagawa, Macromolecules 3 (2) (1970) 109-116.

[3] M. Doi, S.F. Edwards, Journal of the Chemical Society, Faraday Transactions II 74 (1) (1978) 1818-1832.

[4] M. Doi, Journal of Polymer Science Polymer Physics Edition 21 (5) (1983) 667-684.

[5] S.T. Milner, T.C.B. McLeish, Physical Review Letters 81 (3) (1998) 725-728.

[6] R.N. Khaliullin, J.D. Schieber, Macromolecules 43 (14) (2010) 6202-6212.

[7] M. Doi, Journal of Polymer Science, part C - polymer letters 19 (5) (1981) 265-273.

[8] E.V. Menezes, W.W. Graessley, Journal of Polymer Science part B - Polymer Physics 20 (10) (1982) 1817-1833.

[9] G. Marrucci, N. Grizzuti, Gazzetta Chimica Italiana 118 (3) (1988) 179-185.

[10] G. Ianniruberto, G. Marrucci, Journal of Rheology 45 (6) (2001) 1305-1318.

[11] M. Andreev, R.N. Khaliullin, R.J.A. Steenbakkers, J.D. Schieber, Journal of Rheology 57 (2) (2013) 535-557.

[12] T. Yaoita, T. Isaki, Y. Masubuchi, H. Watanabe, G. Ianniruberto G, G. Marrucci, Macromolecules 45 (6) (2012) 2773-2782.

[13] J. Park, D.W. Mead, M.M. Denn, Journal of Rheology 56 (5) (2012) 1057-1081.

[14] Q. Huang, O. Mednova, H.K. Rasmussen, N.J. Alvarez, A.L. Skov, K. Almdal, O. Hassager, Macromolecules 46 (12) (2013) 5026-5035.

[15] Q. Huang, N.J. Alvarez, Y. Matsumiya, H.K. Rasmussen, H. Watanabe, O. Hassager, ACS Macro Letters 2 (8) (2013) 741-744.

[16] H.K. Rasmussen, Q. Huang Q, Journal of Non-Newtonian Fluid Mechanics 204 (1) (2014) 1-6.

[17] R.G. Larson, T. Sridhar, L.G. Leal, G.H. McKinley, A.E. Likhtman, T.C.B. McLeish, Journal of Rheology 47 (3) (2003) 809-818.

[18] K. Osaki, T. Inoue, T. Isomura, Journal of Polymer Science part B - Polymer Physics 38 (14) (2000) 1917-1925.

[19] K. Osaki, T. Inoue, T. Uematsu, Y. Yamashita, Journal of Polymer Science part B - Polymer Physics 39 (14) (2001) 1704-1712.

[20] A.E. Likhtman, T.C.B. McLeish, Macromolecules 35 (16) (2002) 6332-6343.

[21] G.V. Vinogradov, A.Y. Malkin, V.V. Volosevitch, V.P. Shatalov, V.P. Yudin, Journal of Polymer Science: Polymer Physics Edition 13 (9) (1975) 1721-1735.

[22] J.K. Nielsen, O. Hassager, H.K Rasmussen, G.H. McKinley, Journal of Rheology 53 (6) (2009) 1327-1346. 
[24] D.J. Read, K. Jagannathan, S.K. Sukumaran, D. Auhl, Journal of Rheology 56 (4) (2012) 823-873.

[25] G. Liu, H. Sun, S. Rangou, K. Ntetsikas, A. Avgeropoulos, S.-Q. Wang, Journal of Rheology 57 (1) (2013) 89-104.

[26] M.H. Wagner, Rheologica Acta 53 (10-11) (2014) 765-777.

[27] M.H. Wagner, S. Kheirandish, O. Hassager, Journal of Rheology 49 (6) (2005) 1317-1327.

[28] H.K. Rasmussen, Q. Huang, Rheologica Acta 53 (3) (2014) 199-208.

[29] S. Dhole, A. Leygue, C. Bailly, R. Keunings, Journal of Non-Newtonian Fluid Mechanics 161 (1-3) (2009) 10-18.

[30] E. van Ruymbeke, J. Nielsen, O. Hassager, Journal of Rheology 54 (5) (2010) 1155-1172.

[31] K. Yu, H.K. Rasmussen, A.L. Skov, Journal of Rheology 55 (5) (2011) 1059-1067.

[32] C.W. McGuirt, G. Lianis, International Journal of Engineering Science 7 (6) (1969) 579-599.

[33] M. Matsui, D.C. Bogue, Transactions of the Society of Rheology 21 (1) (1977) 453-468.

[34] M. Matsui, D.C. Bogue, Transactions of the Society of Rheology 21 (1) (1977) 133-148.

[35] L.W. Morland, E.H. Lee, Transactions of the Society of Rheology 4 (1) (1960) 233-263.

[36] M. Baumgaertel, A. Schausberger, H.H. Winter, Rheologica Acta 29 (5) (1990) 400-408.

[37] M. Baumgaertel, H.H. Winter, Journal of Non-Newtonian Fluid Mechanics 44 (1) (1992) 15-36.

[38] G. Marrucci, G. Ianniruberto, Macromolecules 37 (10) (2004) 3934-3942.

[39] M. Doi M, S.F. Edwards, The Theory of Polymer Dynamics; Clarendon Press: Oxford (1986).

[40] D. Auhl, J. Ramirez, A.E. Likhtman, P. Chambon, C. Fernyhough, Journal of Rheology 52 (3) (2008) 801-835.

[41] H.K. Rasmussen, J.H. Christensen, S.J. Gøttsche, Journal of Non-Newtonian Fluid Mechanics $93(2-3)(2000)$ 245-263.

[42] A. Bach, K. Almdal, H.K. Rasmussen, O. Hassager, Macromolecules 36 (14) (2003) 5174-5179.

[43] T. Schweizer, J. Meerveld, H.C. Öttinger, Journal of Rheology 48 (6) (2004) 1345-1363.

[44] M.L. Sentmanat, Rheologica Acta 43 (6) (2004) 657-669.

[45] K. Yu, J.M.R. Marín, H.K. Rasmussen, O. Hassager, Journal of Non-Newtonian Fluid Mechanics 165 (1-2) (2010) 14-23.

[46] M.H. Wagner, V.H. Rolón-Garrido, Rheologica Acta 49 (5) (2010) 459-471.

[47] H.K. Rasmussen, Rheologica Acta 54 (1) (2015) 9-18. 
[49] L.J. Fetters, D.J. Lohse, R.H. Colby 'Chain Dimensions and Entanglement Spacings', James E. Mark: Physical Properties of Polymers Handbook, Springer, New York, 2nd ed (2007) 447-455.

[50] Y. Masubuchi, Y. Matsumiya, H. Watanabe, Macromolecules 47(19) (2014) 6768-6775.

[51] T. Sridhar, M. Acharya, D. Nguyen, P.K. Bhattacharjee, Macromolecules 47(1) (2014) 379-386. 


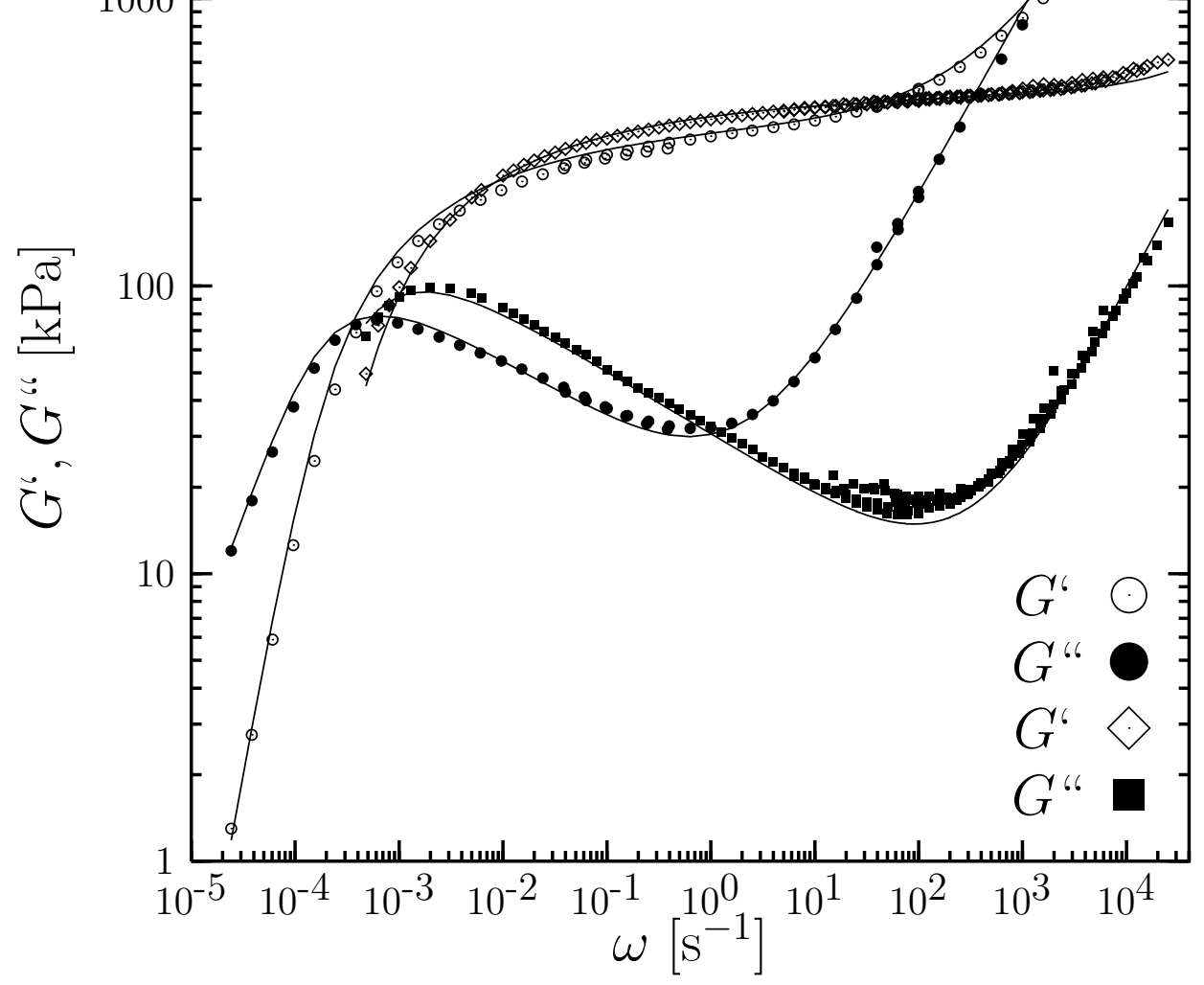

Figure 1: 


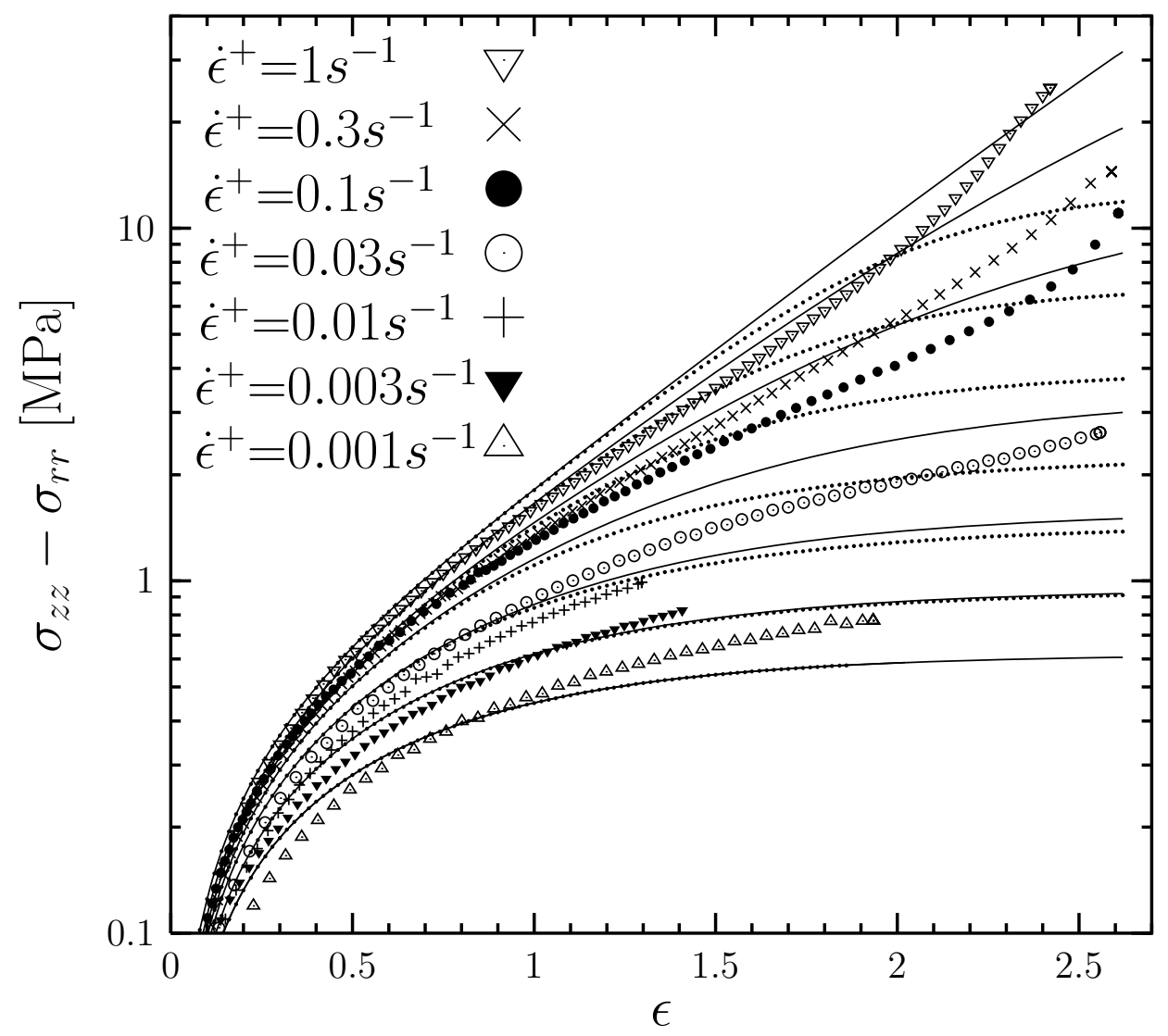

Figure 2: 

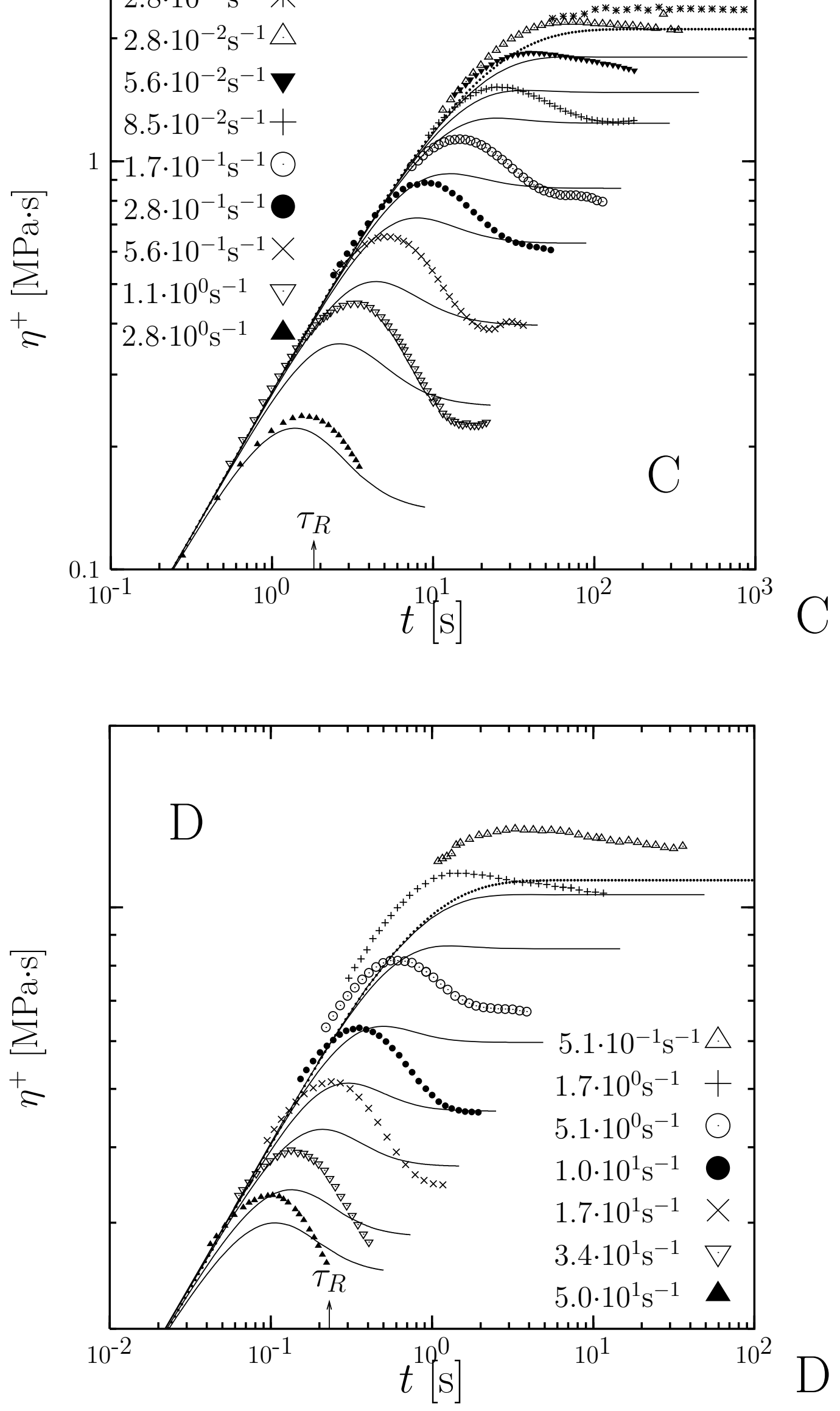